\title{
В.Г. Доронин
}

Днепропетровский госуниверситет

\section{О неравенствах типа Бернштейна для комплекснь тригонометрических полиномов}

Iопуиевы обобиения нсиоторих неравеиств С.Б. Стечкина компхексиозначиые тритонометрические полиномы.

Обозначим через $\mathrm{T}_{2 \mathrm{n+1}}$ множество вещцественных тригонометричес полиномов порядка не более $n_{3}$ а через $\mathrm{T}_{2 \mathrm{n}+1}^{\mathrm{C}}-$ множес комплекснозначньх трнгонометрических полиномов

$$
\theta(t)=\sum_{k=-n}^{n} c_{k} e^{i k t} \quad\left(t \in R, c_{k} \in C, n=0, \pm 1, \pm 2, \ldots\right)
$$

Пусть, как обычно, для $2 \pi$-периодической функции $\mathrm{f}: \mathrm{R} \rightarrow \mathrm{C}$

$$
\text { - }\|f\|_{\infty}=\sup v \text { rai }|f(t)|,\|f\|_{p}=\left\{\int_{0}^{2 \pi}|f(t)|^{p} d t\right\}^{1 / p}
$$

Наконец. пусть

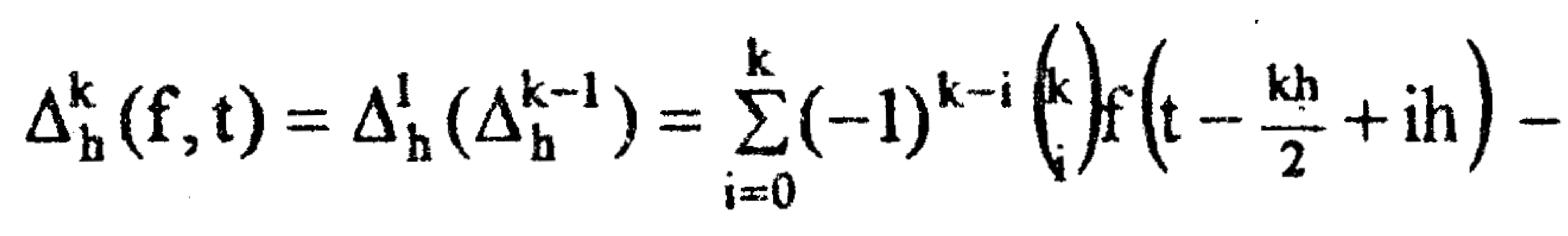

симметричная разность $k$-го порядка функции $f$ в точке $t \mathrm{c}$ шагом $\mathrm{h}$.

Некоторые известные неравенства типа Бернштейна комплексных тригонометрических полиномов представлены в монограф $[1$, гл. 3]. Следуя тем же идеям, в данной работе устанавливается

Теорема. При любых $\mathrm{n}=0,1,2, \ldots, \mathrm{k}=1,2, \ldots$, для люб комплекснозначного тригонометрического полинома $\theta \in \mathrm{T}_{2 \mathrm{n}+1}^{\mathrm{C}} \mathrm{n}$ $0<\mathrm{h}<2 \pi / \mathrm{n}$ и $0<\mathrm{h}<\pi / \mathrm{n}$ соответственно справедливы точн неравенства

$$
\left\|\theta^{(k)}\right\|_{p} \leq k(n, k, h)\left\|\Delta_{h}^{k}(\theta)\right\|_{p} \leq n^{k}\|\theta\|_{p} \quad(1 \leq p \leq \infty)
$$

где

$$
k(n, k, h)=\left(\frac{n}{2 \sin \frac{n h}{2}}\right)^{k}
$$

Эта теорема является обобщением неравенств С.Б. Стечки установившего в [3], что для вещественных тригонометрических полином $\theta \in T_{2 n+1}$ при $p=\infty$ неравенства (1) имеют место.

Доказательство. Докажем первое из неравенств (1), второе доказыва 


\section{- ая аналогично.}

Пусть $\mathrm{p}=\infty$. Как легко убедится, при каждом $\mathrm{t} \in \mathrm{R}$

$$
\begin{gathered}
\mid \theta^{(\mathrm{k})}(\mathrm{t}) \vDash \sqrt{\left(\operatorname{Re} \theta^{(\mathrm{k})}(\mathrm{t})\right)^{2}+\left(\operatorname{Im} \theta^{(\mathrm{k})}(\mathrm{t})\right)^{2}}= \\
=\sup \left\{\alpha \operatorname{Re} \theta^{(\mathrm{k})}(\mathrm{t})+\beta \operatorname{Im} \theta^{(\mathrm{k})}(\mathrm{t}): \alpha^{2}+\beta^{2}=1, \alpha, \beta \in \mathrm{R}\right\}= \\
\left.=\sup \{\alpha \operatorname{Re} \theta(\mathrm{t})+\beta \operatorname{Im} \theta(\mathrm{t}))^{(\mathrm{k})}: \alpha^{2}+\beta^{2}=1, \alpha, \beta \in \mathrm{R}\right\}
\end{gathered}
$$

энем $(\alpha \operatorname{Re} \theta(\mathrm{t})+\beta \operatorname{Im} \theta(\mathrm{t}))^{(\mathbf{k})} \in \mathrm{T}_{2 \mathrm{n}+1}$. Следовательно,

применяя

2мянтое неравенство С.Б. Стечкина, получаем

$$
\begin{aligned}
& \left|\theta^{(\mathrm{k})}(\mathrm{t})\right| \leq \mathrm{k}(\mathrm{n}, \mathrm{k}, \mathrm{h}) \sup \left\{\Delta_{\mathrm{h}}^{\mathrm{k}}(\alpha \operatorname{Re} \theta+\beta \operatorname{Im} \theta) \|_{\infty}: \alpha^{2}+\beta^{2}=1, \alpha, \beta \in \mathrm{R}\right\}= \\
& =\mathrm{k}(\mathrm{n}, \mathrm{k}, \mathrm{h})\left\|\sup \left\{\left(\alpha \Delta_{\mathrm{h}}^{\mathrm{k}}(\operatorname{Re} \theta)+\beta \Delta_{\mathrm{h}}^{\mathrm{k}}(\operatorname{Im} \theta)\right): \alpha^{2}+\beta^{2}=1, \alpha, \beta \in \mathrm{R}\right\}\right\|_{\infty}= \\
& =\mathrm{k}(\mathrm{n}, \mathrm{k}, \mathrm{h})\left\|\sqrt{\left(\Delta_{\mathrm{h}}^{\mathrm{k}}(\operatorname{Re} \theta)\right)^{2}+\left(\Delta_{\mathrm{h}}^{\mathrm{k}}(\operatorname{Im} \theta)\right)^{2}}\right\|_{\infty}=\kappa(\mathrm{n}, \mathrm{k}, \mathrm{h})\left\|\Delta_{\mathrm{h}}^{\mathrm{k}}(\theta)\right\|_{\infty},
\end{aligned}
$$

$\multimap$ и требовалось доказать.

Случай $1 \leq \mathrm{p}<\infty$ исчерпаем на основе приема Стейна. Для любой $2 \pi-$ I.:- бо комплексного тригонометрического полинома $\theta \in \mathrm{T}_{2 n+1}^{\mathrm{C}}$ свертка

$$
(\theta * g)(x)=\int_{0}^{2 \pi} \tau(k-t) g(t) d t \in T_{2 n+1}^{C}
$$

_. -әзедливо неравенство

$$
\left\|(\theta * \mathrm{~g})^{(\mathrm{k})}\right\|_{\infty} \leq \kappa(\mathrm{n}, \mathrm{k}, \mathrm{h})\left\|\Delta_{\mathrm{h}}^{\mathrm{k}}(\theta * \mathrm{~g})\right\|_{\infty} .
$$

Отсюда, из известного экстремального соотношения [2], получаем

$$
\begin{aligned}
& \underbrace{}_{p}=\sup _{\|\varphi\|_{q} \leq 1} \int_{0}^{2 \pi} \theta^{(k)}(v) \varphi(v) d v=\sup _{\|g\|_{q} \leq 1}^{2 \pi} \int_{0}^{(k)}(-t) g(t) d t \leq \sup _{\|g\|_{q} \leq 1}\left\|(\theta * g)^{(k)}\right\|_{\infty} \leq \\
& \leq k(n, k, h) \sup _{\|g\|_{q} \leq 1}\left\|\Delta_{h}^{k}(\theta * g)\right\|_{\infty}=k(n, k, h) \max _{x} \sup _{\|g\|_{q} \leq 1}\left|\left(\Delta_{h}^{k}(\theta) * g\right)(x)\right|= \\
& \quad=k(n, k, h) \max _{x} \sup _{\| g_{l_{q}} \leq 1}\left|\int_{0}^{2 \pi} \Delta_{h}^{k}(\theta)(x-t) g(t) d t\right|=k(n, k, h)\left\|_{h}^{k}(\theta)\right\|_{p},
\end{aligned}
$$

- а требовалось доказать.

\section{Библиографические ссылки}

Э-гейчук Н.П., Бабенко В.Ф., Лигун А.А. Экстремальные свойства полиномов и 二Ейов. К.,1992.

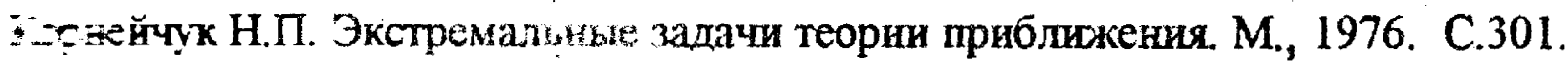

ЕЕлия С.Б. Сбобиение некоторых неравенств С.Н. Бернштейна // Докл. АН СССР. $\therefore 3$. T. 60 , No9. C. $1511-1514$. 